Musées, Patrimoine et Culture scientifiques et techniques

$169 \mid 2017$

janvier-février 2017

\title{
Interroger le rôle de l'espace dans le musée
}

\section{Kali Tzortzi}

\section{OpenEdition}

Journals

Édition électronique

URL : https://journals.openedition.org/ocim/1732

DOI : $10.4000 /$ ocim. 1732

ISSN : 2108-646X

Éditeur

OCIM

Édition imprimée

Date de publication : 1 janvier 2017

Pagination : 12-18

ISSN : 0994-1908

Référence électronique

Kali Tzortzi, «Interroger le rôle de l'espace dans le musée », La Lettre de l'OCIM [En ligne], 169 | 2017, mis en ligne le 01 janvier 2018, consulté le 21 septembre 2021. URL : http://journals.openedition.org/ ocim/1732 ; DOI : https://doi.org/10.4000/ocim.1732

Ce document a été généré automatiquement le 21 septembre 2021.

Tous droits réservés 


\title{
Interroger le rôle de l'espace dans le musée
}

\author{
Kali Tzortzi
}

Au Centre Pompidou (Musée national d'art moderne, Centre de création industrielle, Collections modernes, niveau 5, 2003), le visiteur est invité à explorer les connexions visuelles entre les espaces (a). À la Tate Modern (niveau 3, 2003), des relations visuelles se créent entre les œuvres au sein d'un espace (b).

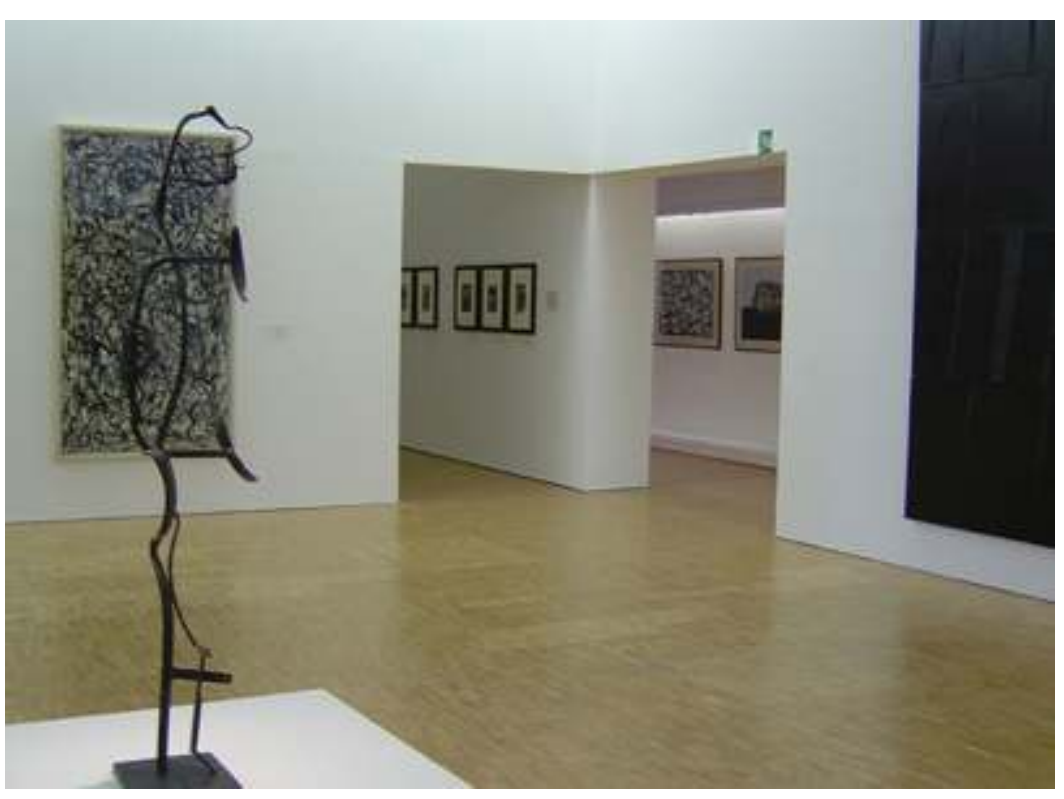




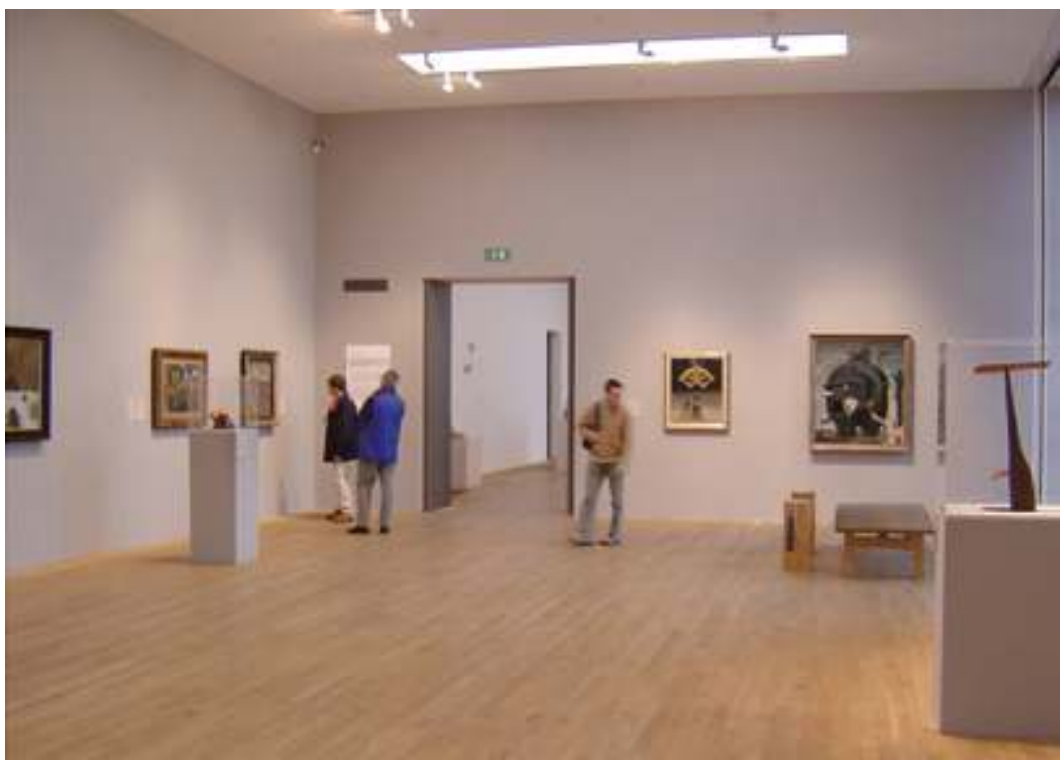

(c) K. Tzortzi, avec l'aimable autorisation du @ Centre Pompidou et de la @ Tate Modern

Depuis quelques décennies, les publications en muséologie accordent une place de plus en plus importante à la dimension spatiale du musée. Parallèlement, la dernière partie du XX ${ }^{e}$ siècle et le début du XXI ${ }^{e}$ sont marqués par une expérimentation et une innovation radicales dans le domaine de l'architecture muséale et de sa morphologie spatiale. Cet article aborde la question à la fois théorique et pratique : comment la manière dont l'espace du musée est organisé influe-t-elle sur l'expérience des visiteurs ? Il souhaite montrer que l'organisation spatiale est d'importance dans un triple sens : elle crée des liaisons entre les salles, structurant l'exploration que nous en faisons ; entre les objets, influençant leur perception et leur lecture ; et entre les visiteurs, affectant leur coprésence et leur interaction. Dans la première partie de l'article, pour analyser les bâtiments-musées comme des systèmes de relations spatiales, nous nous appuyons sur la théorie et la méthodologie de Space syntax. Pour illustrer nos arguments, dans la seconde partie, nous analysons comparativement une série de musées européens spécialement sélectionnés : d'abord, le musée national d'Art moderne - Centre de création industrielle, Centre Pompidou, à Paris, et la Tate Modern, à Londres, qui mettent clairement en lumière deux manières différentes d'organiser l'espace et son expérience, résultant de deux "cultures spatiales" opposées ; et ensuite, l'Ashmolean Museum de l'université d'Oxford, le musée de l'Acropole, à Athènes, et le musée Juif, à Berlin, qui permettent d'explorer la relation entre l'espace et la représentation du temps. Tous ces exemples nourrissent l'idée que l'analyse rigoureuse de la relation entre l'architecture du musée et son caractère spatial, intellectuel et social, peut contribuer à comprendre les expositions d'une manière plus réfléchie et les concevoir plus délibérément.

\section{Des concepts spatiaux dans la littérature muséale : le musée comme ensemble}

2 L'importance de l'espace dans le musée et ses effets sur la manière dont nous percevons l'exposition sont de plus en plus pris en compte par la littérature muséale. Les historiennes d'art Carol Duncan et Rosalind Krauss sont parmi les premières à intégrer la question de l'espace dans le contexte socio-culturel du musée ${ }^{1}$. Elles soutiennent que 
l'organisation de l'espace, en relation avec l'architecture du musée et l'agencement spatial des objets, sont des manifestations d'idéologie et signification sociales et façonnent l'expérience des visiteurs comme un script. Dès lors, ces arguments ont été repris notamment par Mary Anne Staniszewski, Julia Noordegraaf et Victoria Newhouse qui ont étudié des exemples concrets et examiné comment les transformations de la configuration spatiale peuvent refléter des changements dans les idées esthétiques et les concepts culturels ${ }^{2}$. La lecture du musée comme langage et comme texte est une autre approche s'intéressant à la question de l'espace. L'analogie repose sur l'idée que l'exposition fonctionne comme un système de communication, et que "le sens d'une exposition ne survient pas par la seule présence des objets dans un lieu, mais par la disposition, la mise en scène et le recours aux aides qui permettent de les décrypter" ${ }^{3}$. Le concepteur de l'exposition choisit un objet, comme un mot dans un dictionnaire, et celuici, en étant intégré dans l'exposition, "change de statut, devient l'élément d'un ensemble" et sa signification est définie "par les relations qu'il entretiendra avec les autres objets" 4 . Comme le souligne Serge Chaumier, "l'exposition est plus que la somme de ses parties".

3 Un double sens est assigné au texte muséal par la linguiste Louise Ravelli ${ }^{5}$. Dans son ouvrage Museum texts, elle analyse la question des "textes dans les musées", ainsi que celle "des espaces, comme les expositions, qui peuvent être lus et vécus comme des textes significatifs". Selon Ravelli, "une exposition, créée par l'organisation des exposés et des espaces, une sélection et une construction du contenu [...], est un texte significatif : c'est un espace dans lequel les visiteurs déambulent et un espace qu'ils lisent". La théoricienne culturelle Mieke Bal a aussi fait usage de l'analogie avec le langage, en analysant ce qu'elle définit comme le "langage des musées", "parlé" par les éléments de l'exposition comme " la juxtaposition signifiante des objets" et l'architecture ${ }^{6}$. Pour Bal "circuler dans le musée, c'est comme lire un livre", mais il y a un double récit : le récit textuel faisant le lien entre les objets et leurs origines fonctionnelles et historiques ; et le récit spatial dérivant de la "nature séquentielle de la visite".

Jérôme Glicenstein ${ }^{7}$ propose un parallèle entre la mise en relation signifiante des objets exposés et la phase de la disposition en rhétorique. Selon lui, le musée, pour produire son discours, pour "convaincre, toucher [et] persuader", emploie des techniques qui relèvent de differentes parties de la rhétorique antique. Le choix des objets et la définition de leur identité par les historiens d'art et les conservateurs correspondent à la phase de l'invention en rhétorique ("ce qui est à dire"); l'articulation des relations entre les objets et le parcours spatial renvoit à la seconde phase de la disposition ("dans quel ordre le dire"), tandis que la scénographie de l'exposition et l'utilisation des informations textuelles trouvent un écho avec la troisième phase de l'élocution ("comment le dire"). Ainsi, Glicenstein, tout en considérant que l'exposition est une construction d'ordre textuel, met l'accent sur l'idée que la présentation des objets au sein d'une séquence de visite suit une logique qui est liée aux objectifs d'un discours.

5 La question de savoir si l'organisation de l'espace et des objets peut à la fois refléter et créer du sens a été largement abordée par Christopher Whitehead. Les représentations muséales, comme les expositions, sont pour lui "dans un sens une théorie incarnée (embodied theory)". Mais, il ajoute que les musées opèrent théoriquement non seulement pour représenter une discipline ("Les musées ne sont pas des miroirs et leurs représentations ne sont pas simplement une réflexion"), mais aussi pour construire des savoirs, en structurant "des types d'articulation particuliers entre objets et savoirs" 8 . Whitehead propose une analogie spatiale, la comparaison du musée avec la carte dans un 
double sens : l'assemblage dans un musée d'une carte géographique et temporelle des lieux qui sont loin du musée, mais qui sont définis, classés et ordonnés d'une manière théorique ; et le plan du musée, à travers lequel le visiteur fait l'expérience des objets. Dans ce sens, "la carte n'est pas une métaphore pour le musée précisément parce que les cartes et les musées sont constitués de connaissances spatialisées" 9 .

\section{Méthodologie : l'analyse configurationelle}

Ces approches soulignent l'idée que, du point de vue spatial, le musée est un ensemble, un système d'espaces interconnectés. Dans le champ de l'architecture, et plus précisément dans la méthodologie connue sous le nom de Space syntax, on trouve une approche plus analytique du musée comme ensemble, à travers le concept de configuration spatiale. La notion centrale en Space syntax est que la manière dont les bâtiments sont utilisés et fonctionnent ne dépend pas seulement des qualités intrinsèques des espaces mais aussi de leurs interrelations. Des concepts relationnels, comme la connectivité, la profondeur, l' intégration et le choix, sont appliqués à des représentations de l'espace, comme les espaces convexes (où tous les points sont visibles de tous les autres points), les axes et les champs de vue. Cela permet de décrire la configuration spatiale et visuelle du musée telle qu'elle peut être vécue par le visiteur. De cette manière, Space syntax peut aider à parler des aspects "non-discursifs" de l'espace architectural. Dans cet article nous présentons certains concepts fondamentaux proposés par l'analyse configurationelle et nous utilisons des études de cas pour éclairer la manière dont la configuration affecte les fonctions génériques de tous les bâtiments - c'est-à-dire la manière dont ils organisent la circulation et créent différents degrés de co-présence entre leurs utilisateurs - et la fonction spécifique des musées, l'exposition des objets.

\section{Space syntax}

Space syntax est une théorie de l'espace et un ensemble d'outils analytiques, quantitatifs et descriptifs pour analyser les espaces urbains et architecturaux ${ }^{10}$. La mesure syntactique la plus simple, c'est la connectivité, c'est-à-dire l'index du nombre des connexions directes d'un espace. Le contrôle est une autre mesure syntactique qui concerne aussi les relations entre les espaces et leurs voisins immédiats : l'espace qui a plus de contrôle est celui qui a plus de connexions que ses voisins. Mais le concept le plus intéressant du point de vue des musées, c'est la profondeur, la mesure syntactique de la distance topologique (et non métrique) d'un espace à tous les autres espaces d'un ensemble spatial. Un espace peu profond par rapport à tous les autres espaces d'un plan, et donc plus facilement accessible, est un espace intégré. Un espace profond, et donc plus indirectement connecté aux autres espaces, est un espace ségrégué. Le concept d'intégration peut être appliqué aux relations spatiales ou visuelles. À l'aide de l'outil informatique, on assigne des couleurs aux espaces d'après leur profondeur - du rouge pour les mieux intégrés jusqu'au bleu pour les moins intégrés - mettant ainsi en lumière le centre d'intégration en rouge. 
Le graphe du plan du Centre Pompidou (niveau 5) met en lumière les circuits liés à l'axe central (a) et celui de la Tate Modern (niveau 3) révèle un parcours linéaire, formant soit deux séquences spatiales soit une seule plus grande, autour d'un espace central (b).
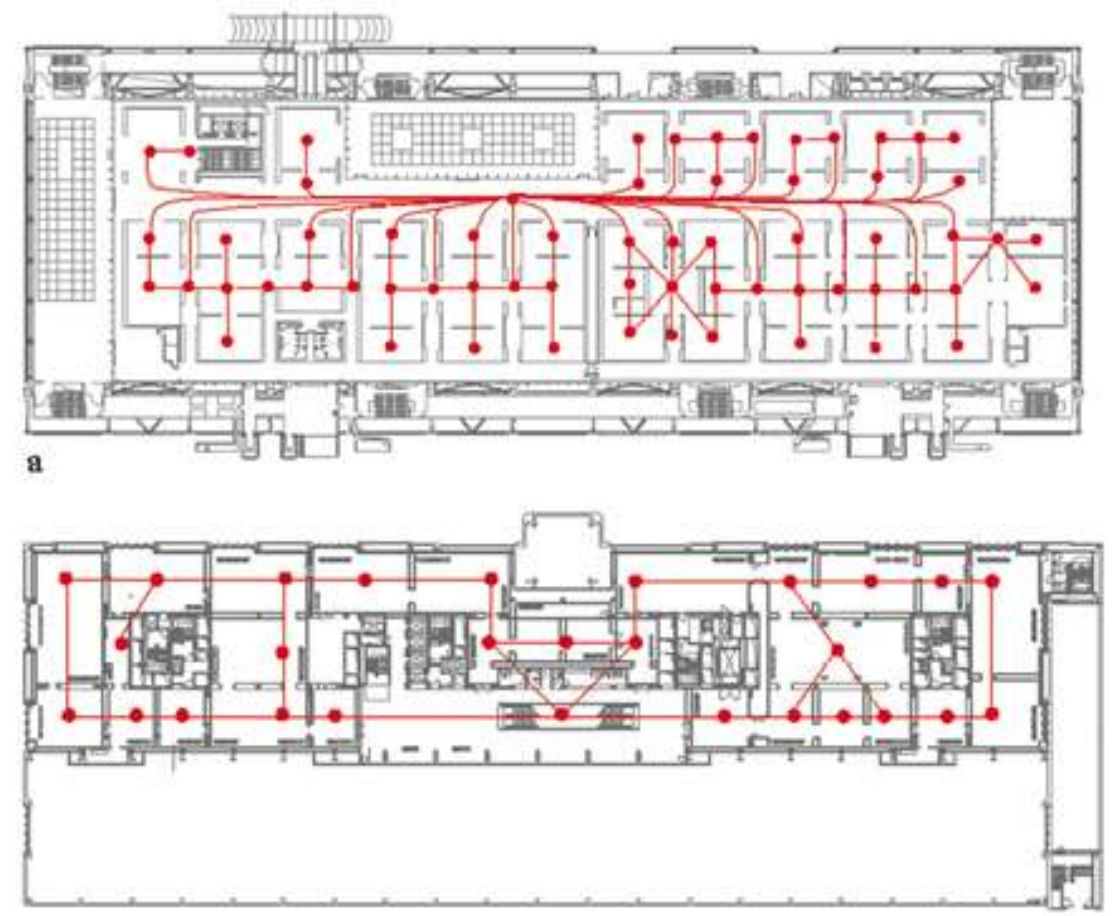

b

(c) K. Tzortzi

\section{La "culture spatiale" du musée}

7 Les premiers exemples analysés, le musée national d'Art moderne, Centre Pompidou (niveau 5) et la Tate Modern (niveau 3) sont des musées nationaux, de grande échelle abrités dans des bâtiments qui ont le rôle de signal urbain ${ }^{11}$. Leurs plans sont caractérisés par des espaces (ou des séquences d'espaces) similaires, organisés en relations géométriques similaires. De plus, tous deux ont une structure tripartite et mettent l'accent sur l'idée d'un axe visuel qui s'étend sur toute la longueur du bâtiment. Mais ce qui les différencie, c'est la manière dont leurs éléments communs sont joints en configuration. On peut montrer cela en utilisant une technique de base de l'analyse configurationelle : le graphe du plan, où les espaces sont représentés par des cercles et leurs liens par des lignes. Au Centre Pompidou, les espaces sont organisés en petits circuits le long de l'axe, offrant plusieurs possibilités de choix de cheminement au niveau local, ce qui signifie que les visiteurs peuvent, en déambulant, faire des choix, changer de direction, changer d'avis. À la Tate Modern, par contre, la structure spatiale procède de l'idée qu'un grand musée nécessite un plan simple ${ }^{12}$. Les salles sont donc beaucoup plus strictement organisées, formant soit deux séquences spatiales soit une seule plus grande, autour d'un espace central. Et là où il $\mathrm{y}$ a du choix, celui-ci tend à conduire rapidement le visiteur à un espace différent de la même séquence, ce qui renforce le caractère déterministe du plan. 
Ces différences entre les deux systèmes spatiaux sont renforcées par leur structure visuelle. La caractéristique principale du Centre Pompidou est la richesse de la visibilité : les connexions denses entre les espaces, dans des directions différentes, génèrent pour le visiteur des relations visuelles qui changent constamment et accentuent un sens dynamique de l'espace. L'organisation visuelle devient ainsi une invitation à déambuler : en offrant une perception simultanée de différentes salles, elle propose des destinations potentielles à découvrir, tout en donnant une impression d'unité au plan compartimenté. À la Tate Modern, en revanche, les connexions entre les espaces sont moins nombreuses et offrent des champs visuels homogènes, principalement dans la direction de la séquence. En utilisant la stratégie opposée aux vues multidirectionelles du Centre Pompidou, la Tate Modern offre une présentation localisée et successive, encourage la concentration, et produit une impression plutôt statique au sein de chaque espace, renforçant ainsi le sens de circuler à travers une séquence d'espaces discrets.

En utilisant l'analyse de l'intégration visuelle du musée, on peut identifier des différences plus complexes. À Pompidou le centre d'intégration visuelle - l'axe central - relie, par des liens visuels et spatiaux fréquents, les salles entre elles, ainsi qu'à l'entrée du musée ; par conséquent, le visiteur peut saisir la structure globale en s'y déplaçant. À la Tate Modern, au contraire, le centre d'intégration visuelle se situe entièrement à la périphérie du plan, et donc le visiteur, une fois dans les salles, a accès à l'information locale seulement. Il ne peut pas se repérer et comprendre intuitivement la structure de l'ensemble du musée.

Mais est-ce que ces différences sont d'importance ? Les différences configurationelles sont en effet reflétées sur la manière dont les visiteurs utilisent l'espace. En observant les parcours des visiteurs, on peut constater qu'au Centre Pompidou, chaque visiteur suit un parcours diffèrent, et la moitié des visiteurs sautent la moitié des salles. Or, leurs itinéraires ont tendance à être individuels et explorateurs. Au contraire, à la Tate Modern, les visiteurs suivent des parcours très similaires, puisque l'itinéraire est pratiquement un cheminement de l'entrée à la fin de la séquence. Mais il y a aussi un effet social qui découle de la manière dont les visiteurs explorent l'espace. Au Centre Pompidou, les visiteurs qui se divisent en directions différentes peuvent se retrouver le long de l'axe. L'organisation du plan maximise les opportunités de rencontres et intensifie le sens d'être co-présent avec d'autres visiteurs. La situation à la Tate Modern est plus simple. Puisque les visiteurs parcourent la même séquence, ils ont tendance à rester avec le même groupe. Donc, l'organisation spatiale de la Tate Modern apparaît efficace - il est peu probable que les visiteurs puissent sauter des salles - mais moins stimulante du point de vue social. 
Des champs de vue créés à partir de points centraux dans trois salles du Centre Pompidou (a) et de la Tate Modern (b). Leur juxtaposition montre clairement le contraste entre les champs de vue créés par le dense réseau des connexions spatiales au Centre Pompidou et ceux à la Tate Modern qui concentrent l'attention du visiteur localement.
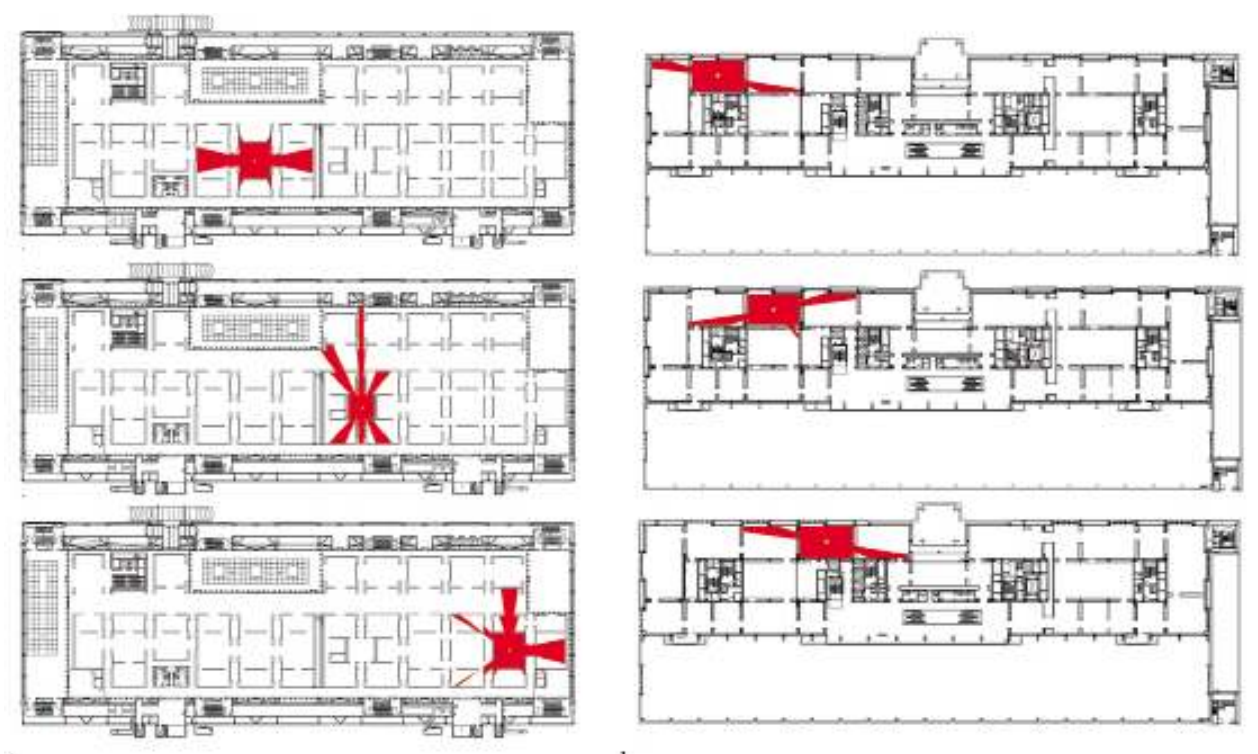

b

\section{(c) K. Tzortzi}

11 À la lumière de ces considérations, nous allons maintenant examiner comment les qualités spatiales des deux musées sont mises en rapport avec la présentation des collections. Au Centre Pompidou, l'accrochage ménage un fil directeur chronologique, et le cheminement organisé en séquences historiques est en synergie avec la configuration spatiale globale structurée et compartimentée. Mais ce qui est plus intéressant, c'est que les propriétés configurationelles - comme l'intégration et le contrôle - ont une influence cruciale sur la manière dont les objets sont perçus et explorés : les oeuvres majeures tendent à être placées dans les espaces qui sont les plus intégrés et ont plus de contrôle, c'est-à-dire soit ceux qui s'ouvrent sur l'axe central, soit ceux qui structurent les axes intérieurs. En même temps, le réseau dense des relations spatiales et visuelles entre les espaces permet un cheminement non-linéaire, encourageant les comparaisons et contrebalançant l'inférence que l'art moderne a évolué le long d'un unique parcours. Regarder un objet au Centre Pompidou signifie découvrir des relations nouvelles, voir la même œuvre dans des combinaisons différentes et percevoir simultanément les réalités visuelles environnantes.

12 À l'inverse de l'accrochage chronologique du Centre Pompidou, la Tate Modern propose une structure anti-narrative et non chronologique qui prend la forme d'un ensemble de salles thématiques, monographiques ou historiques réunies sous un thème conceptuel, visant ainsi à établir des parallèles entre des périodes et montrer des continuités à travers le temps. De même, à la synergie entre l'organisation de l'espace et l'organisation des œuvres de l'exposition qui caractérisent le Centre Pompidou, la Tate Modern oppose leur autonomie mutuelle. Les qualités spatiales, comme les perspectives et les relations visuelles diagonales, ne jouent pas ainsi un rôle majeur pour la perception des œuvres, et les dialogues entre les objets exposés sont limités au sein d'un espace. Ce caractère anti- 
narratif de l'exposition semble au premier regard créer un paradoxe avec la structure spatiale séquentielle. On pourrait dire cependant que cette structure aide à limiter l'attention du visiteur au concept intellectuel de l'exposition tel qu'il est envisagé par les concepteurs. Or, l'originalité du message de l'exposition est soutenue par la fonction contraignante de l'espace.

L'analyse de l'intégration visuelle du plan du Centre Pompidou (a) et de la Tate Modern (b). Dans les deux cas, l'axe central (en rouge) constitue le centre d'intégration visuelle. Au Centre Pompidou l'axe est puissamment connecté aux salles, alors qu'à la Tate Modern les connexions sont plus faibles.
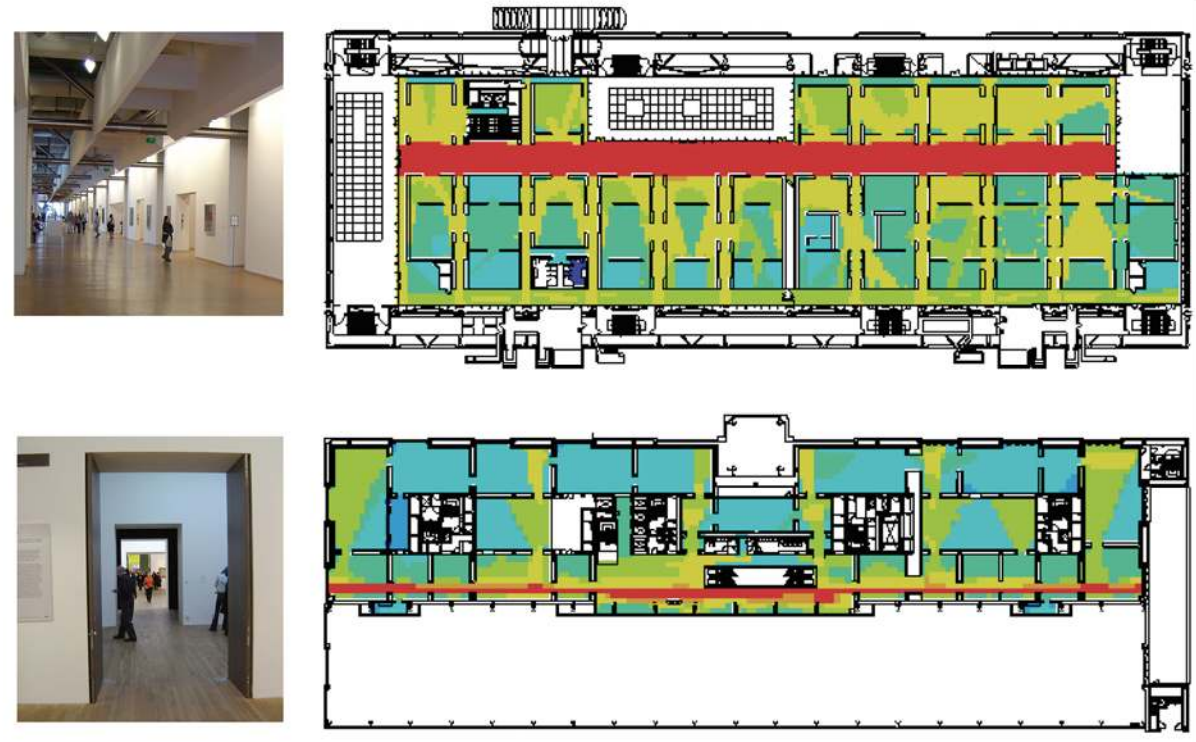

(c) K. Tzortzi, avec l'aimable autorisation du (c Centre Pompidou et de la @ Tate Modern présence et de la perception qu'on a vues, créent la "culture spatiale" différente de chaque musée. À la Tate Modern, l'espace a le rôle primordial d'un outil fonctionnel qui guide la circulation, en facilitant la lisibilité du plan et l'orientation du visiteur, tandis qu'au Centre Pompidou l'espace est systématiquement utilisé comme un dispositif narratif pour suciter des relations entre les œuvres. Étant étroitement liée à l'accrochage, l'organisation spatiale du Centre Pompidou, avec son accent mis sur les choix, les axes et les champs de vue, crée du sens et devient partie de l'esthétique visuelle et donc de l'expérience totale de la visite. À un niveau plus général, on pourrait dire que le Centre Pompidou utilise l'espace pour subvertir l'image temporelle de l'histoire de l'art qu'il présente, alors que la Tate Modern utilise l'espace pour renforcer l'image subversive et atemporelle qu'elle présente.

\section{Des idées spatiales mises en pratique et faisant le lien entre l'espace et le temps}

14 L'analyse configurationelle peut ainsi montrer comment des expériences différentes sont influencées par l'organisation de l'espace, non pas à travers un déterminisme spatial mais comme des effets émergeant de la manière dont les gens perçoivent et utilisent les 
configurations conçues par les programmistes, les architectes et les commissaires d'exposition. Elle peut aussi permettre d'éclairer les manières complexes dont les musées actuels organisent l'espace pour donner de nouveaux sens au discours de l'exposition, comme nous allons le voir dans les exemples suivants qui montrent des illustrations différentes de la relation entre temps et espace muséal.

Le concept "À travers les cultures, à travers le temps" est le thème de l'exposition du nouveau Ashmolean Museum, rouvert en 2009, après avoir été doté de l'extension conçue par Rick Mather. Les collections sont en général organisées par ordre chronologique et par culture, mais elles sont mises en espace de manière à inciter les visiteurs à découvrir non pas les différences, mais les influences, les points de contact et d'échange de techniques et de concepts, reflétant l'idée que les cultures dialoguent, s'influencent mutuellement et partagent une histoire interconnectée. Le développement de cette approche est étroitement lié à l'articulation des espaces et l'utilisation des murs et des balustrades de verre qui relient différentes parties du musée. L'accent est ici mis sur les relations visuelles. L'interconnexion des salles ainsi que les perspectives et les vues à travers plusieurs niveaux attentivement créées, note le directeur Christopher Brown, sont une puissante manifestation du thème de l'exposition, et donc "les relations entre les salles sont souvent aussi importantes que les salles elles-mêmes" ${ }^{13}$.

Vue intérieure de la Tour de l'Holocauste du musée Juif

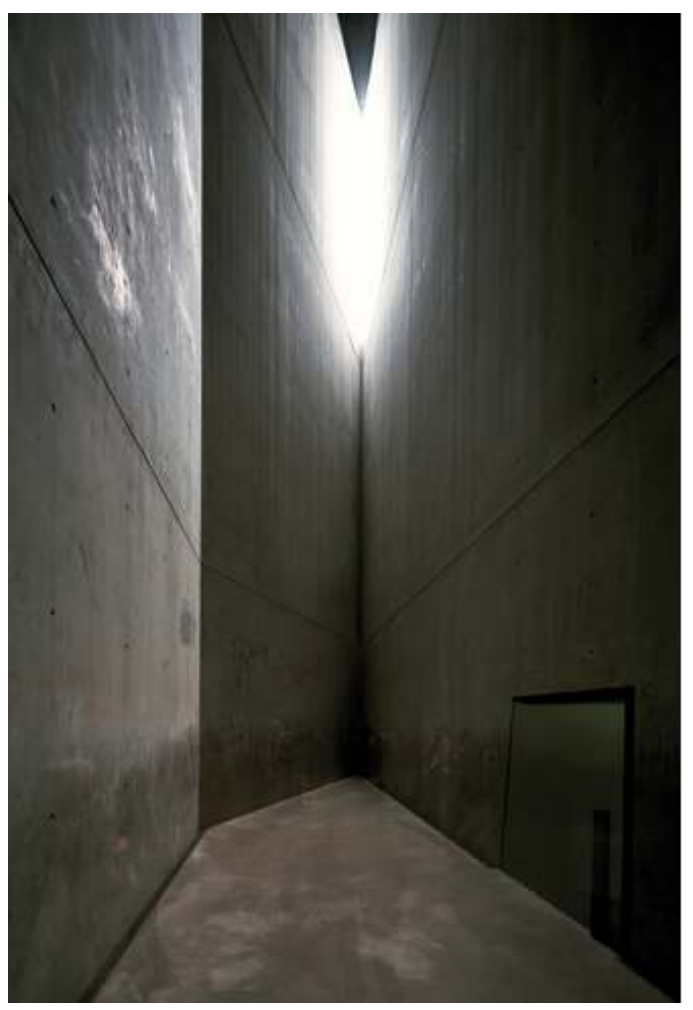

(c) Avec l'aimable autorisation du Studio Libeskind

(c) Bitter Bredt

Une interprétation plus dramatique du temps à travers l'espace est proposée par le musée Juif de Daniel Libeskind, créé en 1999. À la place du concept commun du temps qui se transforme en espace par la succession des salles, le temps se condense en expériences spatiales individuelles, représentant des événements qui se sont déroulés au cours des 
années et des décennies. Ainsi, l'Exil et l'Holocauste sont exprimés par la forme et le contenu d'un seul espace dans chaque cas : dans le premier cas, le Jardin de l'Exil, un espace clos avec quarante-neuf piliers de béton posés sur un sol incliné, qui crée la sensation d'instabilité et d'absence de repères en exil; et dans le second, la Tour de l'Holocauste, un silo vide, de grande hauteur, où la lumière naturelle parvient d'une fente au sommet. Tous deux sont des impasses et les espaces les plus profonds (au sens syntactique) du sous-sol. L'accent normalement mis sur le mouvement des visiteurs est ici remplacé par l'intense expérience locale. La profondeur de ces espaces est accentuée visuellement par une technique architecturale classique qui consiste à créer un axe visuel qui traverse des espaces intermédiaires et aboutit à un espace profond, pour exprimer l'idée du sacré ou du symbolique. C'est en ce sens que les espaces mêmes du bâtiment font partie de l'objet exposé.

Au musée de l'Acropole, des vues traversent quatre niveaux différents : les fondations, la rampe ascendante du rez-de-chaussée, la salle des Caryatides au premier niveau, et la salle du Parthenon au niveau supérieur.

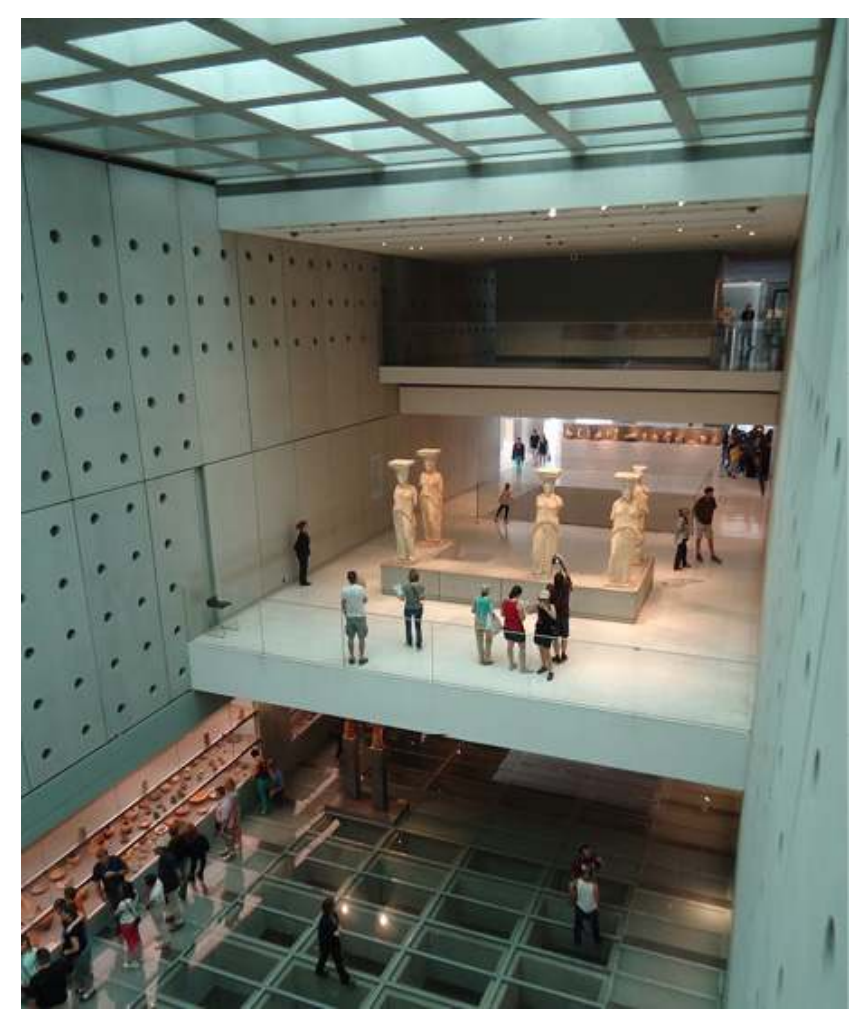

(c) K.Tzortzi, avec l'aimable autorisation du (c) Musée de l'Acropole

17 Un sens spatial est ajouté à celui du temps à travers la forme architecturale du nouveau musée de l'Acropole de Bernard Tschumi, ouvert en 2009. Son architecture a été en grande partie influencée par le site : le musée est bâti au-dessus des vestiges archéologiques de la cité et dans l'environnement immédiat de l'Acropole, et son dernier étage adopte la géométrie et l'orientation du Parthenon. Le visiteur est guidé à travers un parcours chronologique de la Préhistoire jusqu'à l'Antiquité tardive, en montant du rezde-chaussée, qui représente les versants de l'Acropole, au niveau supérieur, consacré au Parthenon. Ainsi le parcours acquiert une fonction symbolique, en termes de temps et d'espace et génère une expérience vécue de la topographie de l'Acropole. Mais, tandis que le discours de l'exposition privilégie la séquence historique, l'espace accentue la 
synchronisation, en limitant les vues d'une salle de l'exposition à l'autre, et donc d'une période historique à l'autre, et en créant des relations visuelles verticales, incluant les vues à travers les sols vitrés et les panoramas extérieurs plus larges, orientés vers les parties contemporaines d'Athènes et vers la cité antique. Ainsi l'espace ajoute au discours de l'exposition une appréhension de l'Acropole plus immédiate et spatiale comme un lieu à travers le temps, plutôt qu'uniquement dans l'histoire. Au musée de l'Acropole, comme au musée Juif, une séquence d'expériences spatiales culmine à l'espace le plus profond, la salle du Parthenon, qui constitue la pièce phare du musée. Ces expériences interagissent avec le discours de l'exposition et constituent ce qu'on peut désigner sous le terme raisonnement spatial de la structure architecturale du musée.

Ashmolean Museum, Université d'Oxford : vue de l'Ashmolean Atrium. Les perspectives entre les salles et entre les niveaux sont des manifestations du thème de l'exposition À travers les cultures, à travers le temps.

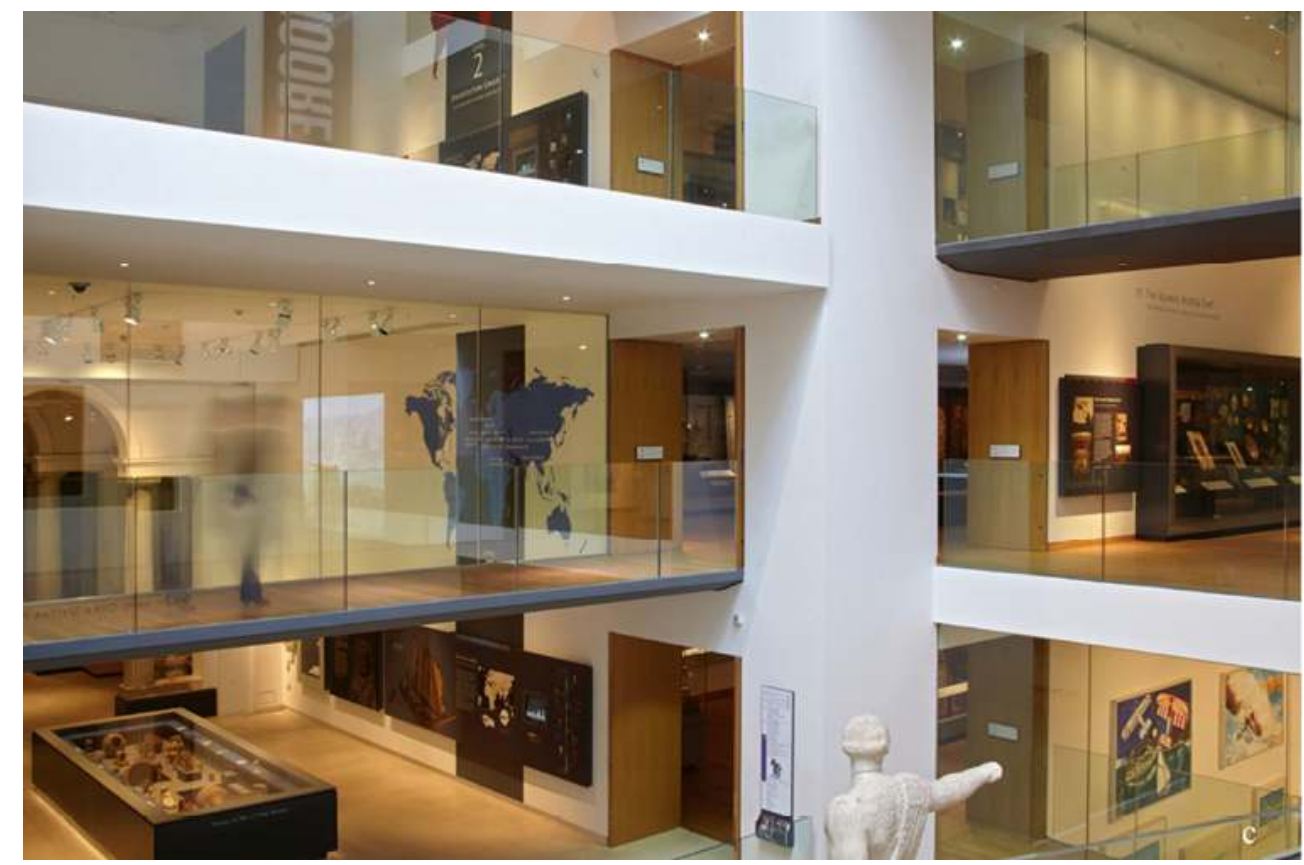

(C) Ashmolean Museum, University of Oxford

\section{Conclusion}

Tous les cas étudiés démontrent l'importance du musée comme ensemble : la manière dont les espaces sont configurés, spécialement à travers les relations visuelles et spatiales, a un effet crucial sur l'expérience du musée et de l'exposition par le visiteur. La forme architecturale et spatiale du musée peut créer un sens d'exploration personnelle ou exprimer une intention plus didactique ; privilégier une expérience spatiale ou transformer la visite à un événement social ; permettre aux visiteurs de voir des relations complexes entre périodes, lieux et objets, ou compléter le contenu de l'exposition plus explicite et rationnel par un mode d'appréhension affectif ${ }^{14}$. Interroger le rôle de l'espace dans le musée et décrire clairement ses dimensions visuelles, kinesthésiques et perceptionelles peut ainsi enrichir théoriquement le design créatif des musées et des expositions. 


\section{NOTES}

1. Duncan, C. Civilizing rituals: inside public art museums. London : Routledge,1995, 192 p. ; Krauss, R. The cultural logic of the late capitalist museum, October, $n^{\circ} 54,1990$, pp. 3-17.

2. Staniszewski, M.-A. The power of display. Cambridge, MA : MIT Press, [1998] 2001, 408 p. ; Noordegraaf, J. Strategies of display: museum presentation in nineteenth- and twentieth-century visual culture. Rotterdam : Museum Boijmans Van Beuningen, 2004, 288 p. ; Newhouse, V. Art and the power of placement. New York : Monacelli Press, 2005, $303 \mathrm{p}$.

3. Chaumier, S. Traité d'expologie : les écritures de l'exposition. Paris: La Documentation française, 2012, $112 \mathrm{p}$.

4. Davallon, J. L'exposition à l'œuvre. Stratégies de communication et médiation symbolique. Paris : L'Harmattan, 1999, 378 p. Voir aussi Desvallées, A., Schärer, M., et Drouguet, N. Exposition, in Desvallées, A., Mairesse, F. et Bergeron, Y. Dictionnaire encyclopédique de muséologie. Paris : Armand Colin, 2011, pp. 133-173.

5. Ravelli, L.-J. Museum texts. Communication frameworks. London ; New York: Routledge, 2006, 200 p.

6. Bal, M. Double exposures : the subject of cultural analysis. New York; London : Routledge, 1996, $352 \mathrm{p}$.

7. Glicenstein, J. La rhétorique au musée, Nouvelle revue d'esthétique $\mathrm{n}^{\circ}$ 6, 2010/2, pp. 177-186.

8. Whitehead, C. Interpreting art in museums and galleries. Abingdon : Routledge, 2012, $216 \mathrm{p}$.

9. Whitehead, C. Museums and the construction of disciplines: art and archaeology in nineteenth century Britain. London : Duckworth, 2009, 160 p.

10. Hillier, B. et Tzortzi, K. Space Syntax : the language of museum space, in Macdonald, Sh. A companion to museum studies, Malden, USA ; Oxford, UK : Blackwell, 2006, pp. 282-301.

11. Pour l'étude complète des musées et l'analyse détaillée de leur conception architecturale et muséographique et du programme scientifique des expositions, voir Tzortzi, K. Museum space : where architecture meets museology. London: Routledge, 2016 [Farnham : Ashgate, 2015], 314 p.

12. Serota, N. The new Tate Gallery of Modern Art, Casabella, n661, 1998, p.14.

13. Brown, C. Introduction, in Ashmolean : Britain's first museum. Oxford : Ashmolean Museum, University of Oxford, 2009, pp. 1-3.

14. Witcomb, A. The materiality of virtual technologies : A new approach to thinking about the impact of multimedia in museums, in Cameron, F. and Kenderline, S. Theorizing digital cultural heritage. A critical discourse. Cambridge, Mass. ; London : MIT Press, 2010, pp. 35-48.

\section{RÉSUMÉS}

Menée à partir de plusieurs études de cas, cette analyse des différentes approches de l'espace dans les institutions muséales, montre comment la forme architecturale et spatiale du musée a des conséquences sur l'expérience de visite des publics et sur la manière dont les visiteurs perçoivent les expositions. 
INDEX

Mots-clés : architecture, public

\section{AUTEUR}

KALI TZORTZI

maître de conférences en muséologie à l'université de Patras et chargée d'enseignement en master de muséologie à l'université d'Athènes

ktzortzi@upatras.gr 